\title{
Potentiation of spinal GABA inhibition as a therapeutic target for chronic neuropathic pain: from transplantation to physical exercise
}

\author{
Emiko Senba ${ }^{1,2}$, Katsuya Kami ${ }^{2,3}$ \\ ${ }^{1}$ Department of Physical Therapy, Osaka Yukioka College of Health Science, Ibaraki City, Osaka, Japan; ${ }^{2}$ Department of Rehabilitation Medicine, \\ Wakayama Medical University, Wakayama City, Wakayama, Japan; ${ }^{3}$ Department of Rehabilitation, Faculty of Wakayama Health Care Science, \\ Takarazuka University of Medical and Health Care, Wakayama City, Wakayama, Japan \\ Correspondence to: Emiko Senba, MD, PhD. Department of Physical Therapy, Osaka Yukioka College of Health Science, 1-1-41 Sojiji, Ibaraki City, \\ Osaka 567-0801, Japan. Email: esenba@wakayama-med.ac.jp. \\ Comment on: Manion J, Khuong T, Harney D, et al. Human induced pluripotent stem cell-derived GABAergic interneuron transplants attenuate \\ neuropathic pain. Pain 2020;161:379-87.
}

Submitted Apr 22, 2020. Accepted for publication Jun 01, 2020.

doi: 10.21037/apm-20-1010

View this article at: http://dx.doi.org/10.21037/apm-20-1010

\section{Chronic neuropathic pain (NPP)}

NPP is induced by damage to the somatosensory system, being the most intractable form among chronic pain diseases. More than 20 million individuals have NPP worldwide and suffer from a great economic burden on individuals and society, and lower quality of life with increased comorbidity such as depression and sleep disturbance (1). NPP is still highly refractive to existing pharmacological treatments. Therefore, chronic pain patients suffer drug tolerance, addiction and various side effects of taking overdoses of analgesics for long time (2). So, there is a highpriority demand to find alternate treatments based on the pathogenesis of NPP syndromes.

Although the etiology of NPP is broad from the periphery to the central nervous system, we focus on peripheral nerve injury-induced NPP in this manuscript. We intend to summarize recent scientific advances in the therapeutic strategies for chronic NPP targeting gammaaminobutyric acid (GABA) interneurons in the spina dorsal horn, trying to find future direction of NPP treatment.

\section{Spinal inhibition in NPP}

GABA is a major inhibitory neurotransmitter in the central nervous system and especially in the spinal dorsal horn. GABA-containing interneurons are thickly distributed in superficial dorsal horn, constituting about $30 \%$ of lamina II neurons. GABAergic inhibitory system has been considered to play a critical role in the pain transmission system.

In order to elucidate the mechanisms of NPP, many studies have focused on spinal dorsal horn neurons. Among these mechanisms the strongest evidence exists for the dysfunction of GABAergic interneurons and altered synaptic transmission of pain signaling pathways in response to peripheral nerve damage. Preclinical studies hitherto have disclosed that peripheral nerve damage induces a selective reduction of GABAergic inhibition in the spinal dorsal horn (3). Apoptotic loss of GABAergic interneurons in the dorsal horn, which was most remarkable 1 week after surgery, has been considered to play a role in the preservation of NPP (4).

More directly, recent work has demonstrated that ablation or hM4Di-mediated silencing of inhibitory interneurons in the spinal dorsal horn causes spontaneous pain and hypersensitivity to thermal and mechanical stimuli (5).

\section{The mechanisms how peripheral nerve injury depresses GABAergic inhibition}

The existing line of evidence suggests the loss of inhibitory 
tone due to NPP can be caused by mechanisms that can be categorized as: (I) decreased number of GABA neurons (apoptosis), (II) down regulation of GABA synthesizing enzyme, glutamic acid decarboxylase (GAD) (epigenetics), (III) decreased GABA release and/or dysfunction of GABA $\mathrm{A} / \mathrm{B}$ receptors, (IV) abnormal cation-chloride cotransporters, such as $\mathrm{K}^{+}-\mathrm{Cl}^{-}$cotransporter-2 (KCC2) in spinal nociceptive neurons. All or some of these may contribute in a concerted manner to the establishment of NPP.

\section{GABA neuron apoptosis}

There has been a controversy about the cause of GABA neuron dysfunction. A question in controversy is whether GABA neurons are lost or only GABA synthesis/release is downregulated.

A recent work has clearly answered to this question, demonstrating that peripheral nerve injury evoked excitotoxic cell death of GABA interneurons in the superficial dorsal horn and contributed to chronic hyperalgesia (6). They clearly showed that elimination of NMDARs fully prevents cell death of GABA interneurons and NPP-like behavior in a mouse model of NPP. The finding that mice lacking proapoptotic Bax are protected against GABA neurons loss strongly indicates the causative relationship of apoptosis and GABA neuron loss. They also noticed that surviving neurons maintain GABA synthesis by means of epigenetics. In line with this, another group has reported that upregulated Cdh1 expression in the trigeminal caudalis nucleus attenuated mechanical allodynia by reducing apoptosis of GABA interneurons caused by chronic constriction injury (CCI) of infraorbital nerve. The Cdh1 inhibition is thought to trigger apoptotic cell death.

Taken together, these studies clearly show that peripheral nerve damage induced apoptosis of GABAergic neurons is the primary cause of NPP in animal models.

\section{KCC2 reduction in nociceptive neurons}

Another mechanism of NPP is a reduction of GABAergic inhibition due to a depolarizing shift in chloride equilibrium, which is induced by a $50 \%$ reduction of KCC2 expression in dorsal horn neurons following peripheral nerve injury. KCC2 is a transporter which maintains intracellular $\mathrm{Cl}^{-}$concentration at low level (7). Reduction of KCC2 in dorsal horn neurons have been demonstrated in NPP model animals (8), suggesting that reduction of KCC2 expression seriously affects the potency of GABAergic inhibition. GABAergic interneurons receiving A-beta inputs and supplying feed-forward inhibition to nociceptive lamina I projection neurons may paradoxically cause excitation of these projection neurons when KCC2 contents of these neurons are downregulated.

\section{Therapeutic approaches using a surgical procedure}

Given the established role of GABAergic dysfunction in the etiology of NPP, restoration of this function represents an attractive and promising therapeutic approach. At least three approaches targeting spinal cord using a surgical procedure have been attempted in preclinical and clinical studies: (I) intrathecal injection of GABA mimetic drugs, (II) spinal cord stimulation and (III) cell transplantation.

\section{Intrathecal injection}

Since Eaton et al. (9) reported that intrathecal injection of GABA in an animal model of NPP permanently reversed NPP symptoms, a variety of analgesics, including GABA $\mathrm{A} / \mathrm{B}$ agonists, and even less studied agents has been tried with novel gene-based intrathecal targeting methodologies. However, as Eaton et al. (9) has reported, when GABA was administered any later than 2-3 weeks after nerve injury, it was unsuccessful to prevent such NPP symptoms, indicating that this approach is not suitable for chronic NPP.

\section{Spinal cord stimulation (SCS)}

After 40 years of clinical practice, it has been proved that SCS is an accepted and cost-effective therapy for the treatment of intractable chronic pain. Preclinical and clinical studies that used high frequency-SCS for a variety of chronic pain syndromes have been reported. The effects of SCS on the spinal GABAergic neurons have been studied in a rat model of NPP (10). In animals that respond to SCS, a significant increase of GAD 65 was observed in superficial dorsal horn, probably reflecting an increased GABA production following SCS. Recently, López-Álvarez et al. (11) used a rat model of sciatic nerve transection and repair to show that chronic stimulation protocols restored the amount of $\mathrm{KCC} 2$, which had been significantly decreased by nerve injury, in the dorsal horn of these rats. 


\section{Cell transplantation of GABAergic interneurons}

A different approach to augmenting inhibitory tone, spinal transplantation of GABA neurons, may be a promising NPP therapy. The idea of transplanting cells that secret GABA or GABAergic neurons directly into the spinal cord of NPP models has been paid much attention.

This approach was first demonstrated in rodent models in which chronic pain behavior was attenuated by grafting GABAergic neurons or cells modulated to secrete GABA by bioengineering $(12,13)$. Then, fetal mouse GABAergic cells or human neural precursor cells (NPCs) differentiated to show a GABAergic phenotype were transplanted into the spinal cord 10 days after spinal nerve ligation surgery (14). The transplants successfully attenuated allodynia induced by peripheral nerve injury.

In the next stage, immature mouse telencephalic GABA progenitor cells were transplanted into the spinal cord of NPP model animals. Jergova $e t$ al. induced NPP in rats via CCI of the sciatic nerve, then grafted GABAergic precursor cells developed from E14 lateral ganglionic eminence (LGE) cells into the ipsilateral lumbar spinal cord 1 week after the constriction injury (15).

Another study examined the effects of transplanting of GABAergic progenitors from E12.5-13.5 mouse medial ganglionic eminence (MGE) into the spinal cord of mice manifesting NPP due to spared sciatic nerve injury (16). Only $1-3 \%$ of grafted cells survived but they could differentiate into GABAergic neurons, constructing significant functional contacts with both primary afferent fibers and dorsal horn neurons. The density of GABA producing enzymes GAD65/67 was also increased in the spinal cord, and mice received these grafts showed a complete loss of mechanical allodynia due to peripheral nerve damage.

In another study from the same group, GABAergic cell grafting in a paclitaxel induced chemotherapy model of NPP showed beneficial effects. In this study, transplanting of E12.5-13.5 mouse MGE progenitors into the spinal cord 1 week after the development of hyperalgesia attenuated NPP symptoms. Interestingly, such symptoms were not attenuated when MGE cells from mice lacking vesicular GABA transporter (VGAT) gene were grafted, indicating that anti-hyperalgesic effects of MGE cell transplantation are dependent on GABA release by grafted interneurons.

Although these previous studies have demonstrated that fetal GABA neuron progenitors can effectively reduce NPP, the multipotent nature of these progenitor cells makes them unsuitable for clinical application. Multipotent MGE progenitors can differentiate into other cell types, therefore elevating the risk of cancer generation.

Here, Manion et al. (17) has recently overcome this problem by showing that terminally differentiated human induced pluripotent stem cell-derived GABAergic (iGABAergic) neurons transplanted into the spinal cord significantly reduced NPP symptoms of mice subjected to spinal nerve injury. They found that iGABAergic neurons can survive in the spinal cord of NPP mice, and these cells were able to alleviate pain long term $(\sim 2$ months $)$ with a single treatment, demonstrating that this translational strategy is a viable option for the alleviation of established NPP. Differentiated iGABAergic neurons can be used for autologous transplants and it is obvious that this approach has far greater clinical relevance than previous ones.

However, the only caveat, if any, of this approach is that it can be applied to only a limited number of patients because it requires advanced technology and equipment, in addition to a great health care cost.

\section{Non-surgical approach: physical exercise therapy}

In this context, recently non-surgical and cost-effective procedure was found to retrieve the reduced GABAergic inhibition in the nerve-injured spinal cord. We demonstrated using mice subjected to partial sciatic nerve ligation (PSL) that treadmill running effectively blocked PSL-induced decrease of GABA and GAD65/67 immunoreactivities, leading to the preservation of GABA in neurons and neuropils in the dorsal horn of these animals (18) (Figure 1A). Mechanical hypersensitivity due to PSL was significantly reduced in PSL-Runner mice compared to that in PSLsedentary mice. Thresholds of pain behavior positively correlated with GABA and GAD65/67 levels and numbers of GABA interneurons in the ipsilateral dorsal horn of PSLRunner mice. Thus, we concluded that the improvement of impaired GABAergic inhibition constitutes a new mechanism that explains exercise-induced hypoalgesia (EIH).

Effectiveness of physical exercise in attenuating NPP has been established in preclinical and clinical researches. Many studies have attempted to clarify the underlying mechanisms of EIH at various levels of peripheral and central nervous systems [for review see (19)].

The cutting-edge therapy of transplantation surgery will be applied to only a limited number of patients, while physical exercise is a first-line therapy for every patient 
A

A
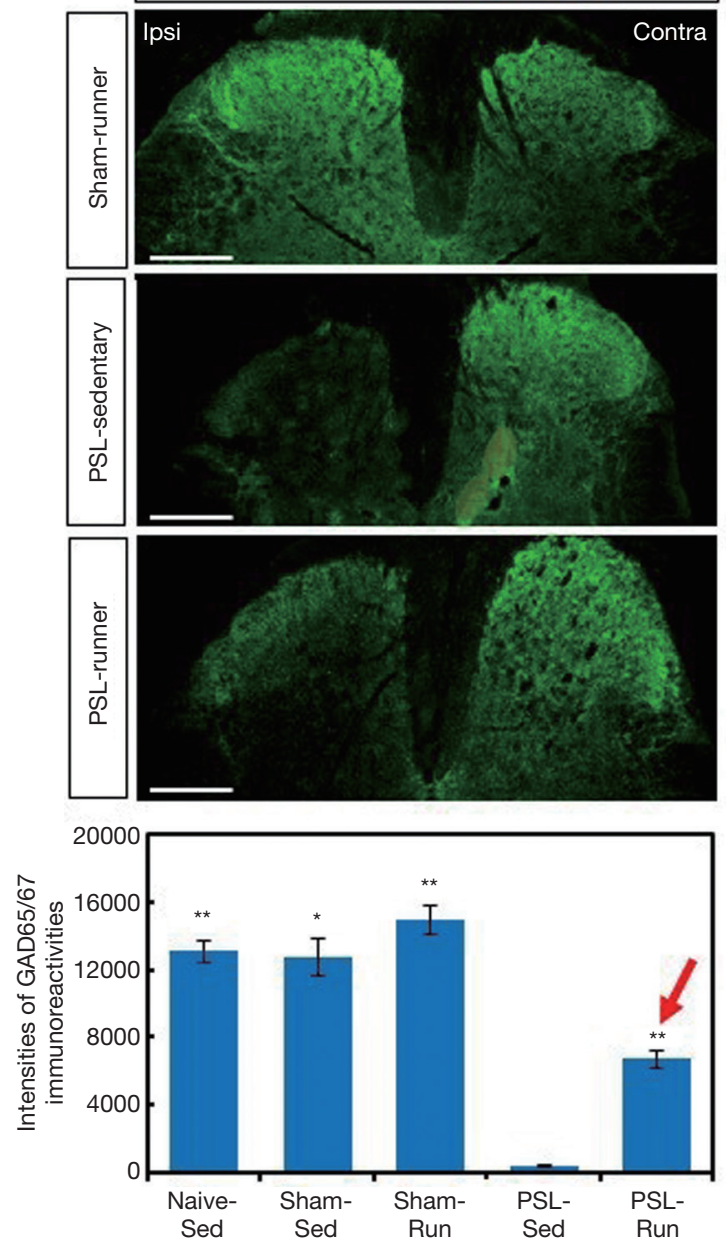

B
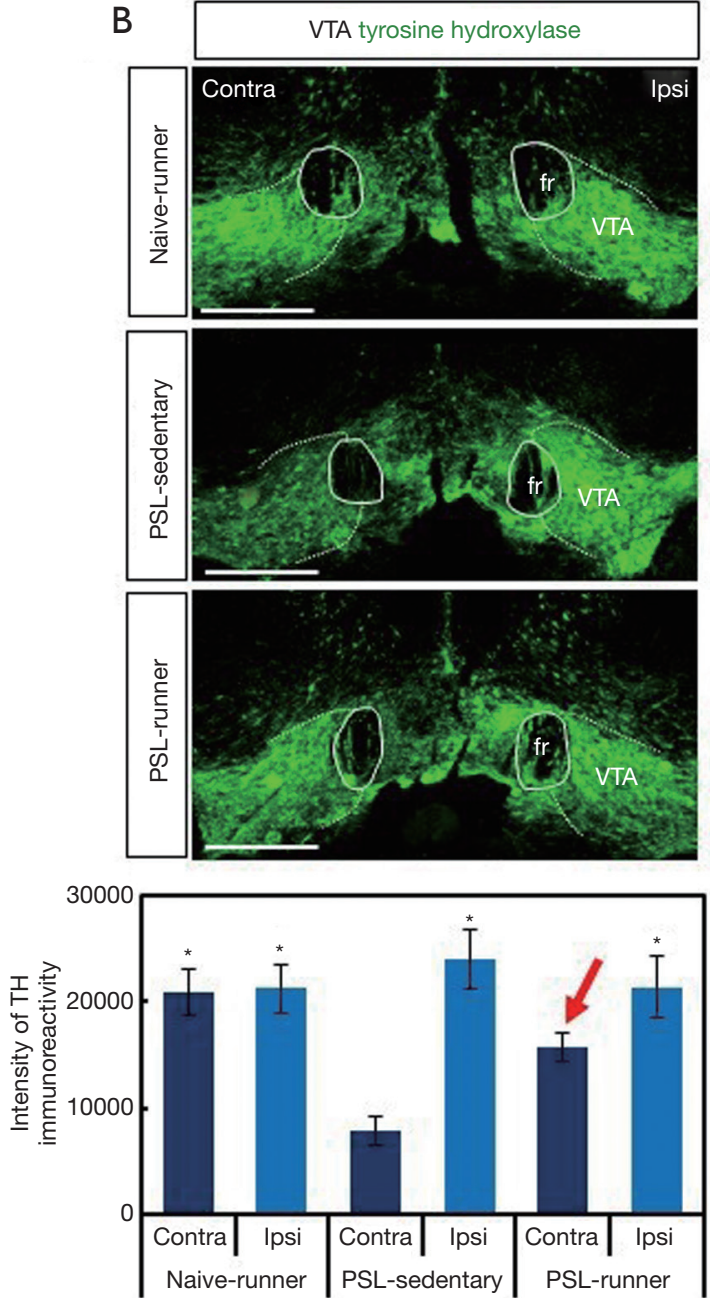

Figure 1 Effects of exercise on spinal GABA synthesis and dopamine synthesis in the VTA. (A) PSL reduced GAD65/67 immunoreactivities in the ipsilateral (ipsi) dorsal horn (L4-5) of PSL-sedentary mice, but exercise by treadmill running prevented it in PSL-Runner mice (indicated by an arrow). Scale bar: $200 \mu \mathrm{m}$. A bar chart shows that GAD65/67 immunoreactivity was significantly weaker in PSL-Sedentary mice compared to the other groups. The significance of differences among groups was calculated by a one-way ANOVA and Tukey's post hoc test (vs. PSL-sedentary; *, $\mathrm{P}<0.01$; ${ }^{* *}, \mathrm{P}<0.001 ; \mathrm{n}=6$ ); (B) TH immunoreactivity in the VTA was decreased by PSL surgery in PSLsedentary mice but voluntary running restored it in PSL-Runner mice (indicated by an arrow). fr: fasciculus retroflexus, Scale bar: $300 \mu \mathrm{m}$. A bar chart shows that TH-immunoreactivities were significantly augmented by voluntary wheel running, while TH-immunoreactivities were significantly decreased in the contralateral (contra) side of the lateral VTA in PSL-sedentary mice compared to the other groups ( $v s$. PSL-sedentary contra; *, $\mathrm{P}<0.01 ; \mathrm{n}=5$ ). Quantified data are presented as the mean $\pm \mathrm{SEM}$. GABA, gamma-aminobutyric acid; VTA, ventral tegmental area; PSL, partial sciatic nerve ligation; GAD, glutamic acid decarboxylase; ANOVA, analysis of variance; TH, tyrosine hydroxylase; SEM, standard error of the mean.

suffering chronic pain. Physical exercise is a safe and costeffective therapy because it does not require any surgical procedure, any special equipment and can be carried out in anywhere in the world. More recently we have elucidated that physical exercise not only reduces pain by restoring GABAergic inhibition in the spinal cord (18), but also activates brain reward system, i.e., dopaminergic neurons in the ventral tegmental area (VTA), restoring dopamine synthesizing enzyme tyrosine hydroxylase $(\mathrm{TH})$, which was decreased by PSL surgery $(20,21)$ (Figure 1B).

Voluntary exercise has been considered as a strong natural reward, and the activated mesolimbic reward system 


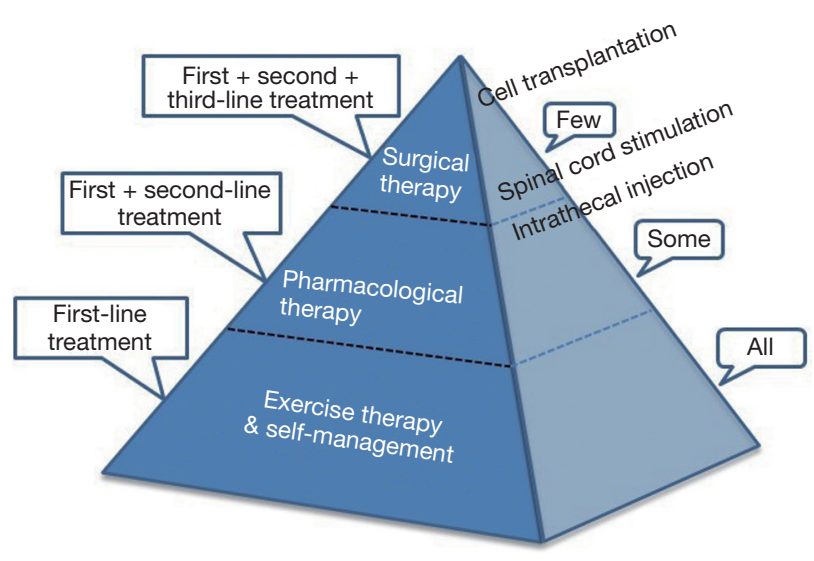

Figure 2 Treatment pyramid for NPP targeting spinal GABA neurons. All patients should be proposed exercise and selfmanagement as first-line treatment, while some will need drug therapy as second-line treatment, and few will need surgical interventions including cell transplantation as third-line treatment [modified from Figure 1 of ref (25)]. NPP, neuropathic pain; GABA, gammaaminobutyric acid.

may play a part in suppression of tonic pain. As a matter of fact, we demonstrated that voluntary exercise increases the expression of phosphorylated CRE binding (pCREB) protein in VTA dopamine neurons, which would enhance the transcription of $\mathrm{TH}$ gene and then dopamine synthesis, thereby contributing to the activation of the mesolimbic reward pathway in a mouse model of NPP. Voluntary running in PSL mice on a running wheel equipped in each cage for 15 days after PSL surgery remarkably attenuated NPP behaviors compared to those of PSL-sedentary mice and the recovery was in proportion to the total running distance and the levels of pCREB and TH in the VTA of these mice. Activation of brain reward system may also improve depression and sleep disturbance, and eventually improve patients' quality of life. We further elucidated the mechanisms how voluntary exercise can activate VTA dopamine neurons; glutamatergic /cholinergic neurons in the latero-dorsal tegmental nucleus and orexin neurons in the lateral hypothalamic area are activated by exercise and then project to the VTA (21).

Not only the spinal mechanisms, but also extensive plasticity and sensitization of the central nervous system have been implicated in the etiology of chronic NPP. Therefore, chronic pain patients were shown to suffer severe dysfunction in various brain regions involved in perception, emotion, and cognition, and eventually in pain behavior (22). Cell transplantation approach mainly targets spinal mechanisms, while physical exercise can influence more extensive central brain regions, especially regions mediating pain emotion, in which cortico-limbic system and meso-limbic reward system play pivotal roles. It has been demonstrated that chronic NPP causes dysfunction of cortico-limbic system, such as medial prefrontal cortex, amygdala, nucleus accumbens and hippocampus (23), and VTA dopamine neurons are inhibited via activated glutamatergic inputs from the lateral habenula. Recently we have demonstrated that physical exercise can effectively counteract these chronic NPP-induced dysfunctions and inhibition (Kami et al., in submission). Through these central mechanisms, physical exercise can exert both analgesic and emotional (anti-depressive) influence on chronic pain patients, eventually and effectively improving their quality of life.

\section{Future prospect: NPP treatment strategy}

Various surgical and non-surgical therapeutic approaches, targeting the potentiation of GABA interneurons in the spinal cord have been reviewed based on recent publications, focusing on the possibility of future clinical application. Especially, spinal transplantation of iGABAergic cells and physical exercise seem to be most practical and promising, from a viewpoint of safety and effectiveness.

To fully understand and utilize these treatment strategies, mechanistic and translational investigations will be essential, with intimate communication between basic science and clinical researchers for the better experimental designs and clinical trials, to optimize the potential of these therapeutic technologies. It should be emphasized that these two strategies are not conflicting in nature, but mutually complementing, as shown in a recent study that physical exercise and GABAergic neuronal progenitor cell transplants showed mutually beneficial effects in a rodent model of spinal cord injury (24).

The exercise therapy can be carried out alone or combined with pharmacotherapy and surgical treatment. Figure 2 shows the NPP treatment pyramid, a principle strategy of therapies targeting spinal GABA interneurons, modified from that proposed for osteoarthritis treatment (25). NPP will be best controlled using education and exercise, which requires an active lifestyle alteration by the patient. Exercise and self-management are not only effective but also cost-effective. In a meta-analysis study in which the 
cost-effectiveness of first-line treatments were investigated, exercise therapy was assessed to be most cost saving. All the NPP patients should be proposed to exercise as first-line treatment, and drug therapy and surgical intervention will be added when it is necessary. Patients who need secondline treatment might have a fear of dependency on analgesic drugs such as opioids, but the knowledge of the safe and effective third-line therapy will give them more confidence and hope. We hope that the evidence-based information will help patients and medical staffs to communicate each other for the best practice treatments. Multidisciplinary, patient-centered and stepped care strategy is essential for the execution of such clinical guidelines and the selection of suitable non-surgical or surgical treatment options for chronic pain patients.

\section{Acknowledgments}

Funding: The authors' EIH studies were supported by research grants from KAKENHI [Grants-in-Aid for Scientific Research (C) 15K01427 and 15K08677 from the Japan Society for the Promotion of Science].

\section{Footnote}

Provenance and Peer Review: This article was commissioned by the editorial office, Annals of Palliative Medicine. The article did not undergo external peer review.

Conflicts of Interest: Both authors have completed the ICMJE uniform disclosure form (available at http://dx.doi. org/10.21037/apm-20-1010). ES and KK report grants from the Japan Society for the Promotion of Science, during the conduct of the study.

Ethical Statement: The authors are accountable for all aspects of the work in ensuring that questions related to the accuracy or integrity of any part of the work are appropriately investigated and resolved.

Open Access Statement: This is an Open Access article distributed in accordance with the Creative Commons Attribution-NonCommercial-NoDerivs 4.0 International License (CC BY-NC-ND 4.0), which permits the noncommercial replication and distribution of the article with the strict proviso that no changes or edits are made and the original work is properly cited (including links to both the formal publication through the relevant DOI and the license).
See: https://creativecommons.org/licenses/by-nc-nd/4.0/.

\section{References}

1. Senba E. A key to dissect the triad of insomnia, chronic pain, and depression. Neurosci Lett 2015;589:197-9.

2. Finnerup N B, Haroutounian S, Kamerman P, et al. Neuropathic pain: an updated grading system for research and clinical practice. Pain 2016;157:1599-606.

3. Ibuki T, Hama AT, Wang XT, et al. Loss of GABA immunoreactivity in the spinal dorsal horn of rats with peripheral nerve injury and promotion of recovery by adrenal medullary grafts. Neuroscience 1997;76:845-58.

4. Moore KA, Kohno T, Karchewski LA, et al. Partial peripheral nerve injury promotes a selective loss of GABAergic inhibition in the superficial dorsal horn of the spinal cord. J Neurosci 2002;22:6724-31.

5. Koga K, Kanehisa K, Kohro Y, et al. Chemogenetic silencing of GABAergic dorsal horn interneurons induces morphine-resistant spontaneous nocifensive behaviours. Sci Rep 2017;7:4739.

6. Inquimbert $\mathrm{P}, \mathrm{Moll} \mathrm{M}$, Latremoliere A, et al. NMDA receptor activation underlies the loss of spinal dorsal horn neurons and the transition to persistent pain after peripheral nerve injury. Cell Rep 2018;23:2678-89.

7. Coull JA, Boudreau D, Bachand K, et al. Trans-synaptic shift in anion gradient in spinal lamina I neurons as a mechanism of neuropathic pain. Nature 2003;424:938-42.

8. Li L, Chen SR, Chen H, et al. Chloride homeostasis critically regulates synaptic NMDA receptor activity in neuropathic pain. Cell Rep 2016;15:1376-83.

9. Eaton MJ, Martinez MA, Karmally S, et al. A single intrathecal injection of GABA permanently reverses neuropathic pain after nerve injury. Brain Res 1999;835:334-9.

10. Ultenius C, Song Z, Lin P, et al. Spinal GABAergic mechanisms in the effects of spinal cord stimulation in a rodent model of neuropathic pain: is GABA synthesis involved? Neuromodulation 2013;16:114-20.

11. López-Álvarez VM, Cobianchi S, Navarro X. Chronic electrical stimulation reduces hyperalgesia and associated spinal changes induced by peripheral nerve injury. Neuromodulation 2019;22:509-18.

12. Eaton MJ, Plunkett JA, Martinez MA, et al. Transplants of neuronal cells bioengineered to synthesize GABA alleviate chronic neuropathic pain. Cell Transplant 1999;8:87-101.

13. Stubley LA, Martinez MA, Karmally S, et al. Only early intervention with gamma-aminobutyric acid cell therapy is 
able to reverse neuropathic pain after partial nerve injury. J Neurotrauma 2001;18:471-7.

14. Mukhida K, Mendez I, McLeod M, et al. Spinal GABAergic transplants attenuate mechanical allodynia in a rat model of neuropathic pain. Stem Cells 2007;25:2874-85.

15. Jergova S, Hentall ID, Gajavelli S, et al. Intraspinal transplantation of GABAergic neural progenitors attenuates neuropathic pain in rats: a pharmacologic and neurophysiological evaluation. Exp Neurol 2012;234:39-49.

16. Bráz JM, Sharif-Naeini R, Vogt D, et al. Forebrain GABAergic neuron precursors integrate into adult spinal cord and reduce injury-induced neuropathic pain. Neuron 2012;74:663-75.

17. Manion J, Khuong T, Harney D, et al. Human induced pluripotent stem cell-derived GABAergic interneuron transplants attenuate neuropathic pain. Pain 2020;161:379-87.

18. Kami K, Taguchi Ms S, Tajima F, et al. Improvements in impaired GABA and GAD65/67 production in the spinal dorsal horn contribute to exercise-induced hypoalgesia in a mouse model of neuropathic pain. Mol Pain 2016;12:1744806916629059.

Cite this article as: Senba E, Kami K. Potentiation of spinal GABA inhibition as a therapeutic target for chronic neuropathic pain: from transplantation to physical exercise. Ann Palliat Med 2020;9(5):2430-2436. doi: 10.21037/apm-20-1010
19. Kami K, Tajima F, Senba E. Exercise-induced hypoalgesia: potential mechanisms in animal models of neuropathic pain. Anat Sci Int 2017;92:79-90.

20. Senba E, Kami K. A new aspect of chronic pain as a lifestyle-related disease. Neurobiol Pain 2017;1:6-15.

21. Kami K, Tajima F, Senba E. Activation of mesolimbic reward system via laterodorsal tegmental nucleus and hypothalamus in exercise-induced hypoalgesia. Sci Rep 2018;8:11540.

22. Bushnell MC, Ceko M, Low LA. Cognitive and emotional control of pain and its disruption in chronic pain. Nat Rev Neurosci 2013;14:502-11.

23. Thompson JM, Neugebauer V. Cortico-limbic pain mechanisms. Neurosci Lett 2019;702:15-23.

24. Dugan EA, Jergova S, Sagen J. Mutually beneficial effects of intensive exercise and GABAergic neural progenitor cell transplants in reducing neuropathic pain and spinal pathology in rats with spinal cord injury. Exp Neurol 2020;327:113208.

25. Roos EM, Juhl CB. Osteoarthritis 2012 year in review: rehabilitation and outcomes. Osteoarthritis Cartilage 2012;20:1477-83. 